\title{
Late Onset Sepsis and Incomplete Kawasaki Disease in a 3-Month-Old Infant: A Case Report and Literature Review
}

\author{
Suraj Pratap ${ }^{\mathrm{a}, \mathrm{d}}$, Damodar R. Thambireddy ${ }^{\mathrm{b}}$, Hermann Mendez ${ }^{\mathrm{c}}$
}

\begin{abstract}
Despite advances in the antimicrobial therapy and implementation of a multitude of screening protocols, group B streptococcus (GBS) infection remains a major cause of morbidity and mortality in newborns and young infants. The incidence of GBS septicemia, however, declines drastically with age, and it is rarely implicated as a cause of sepsis in infants older than 2 months. Recently we were involved in caring for a female infant from Brooklyn who presented with subtle signs of infection and her blood culture later grew GBS. She also had significant coronary artery dilatation suggestive of another ongoing inflammatory process which could not be justified solely by a GBS infection. Kawasaki disease (KD) is an acute febrile illness of childhood characterized by self-limited vasculitis and is known to cause coronary artery changes if not treated timely. The first case of this unusual illness was observed by Tomisaku Kawasaki in 1961 in a 4-year-old child who presented with an unremitting fever and a rash. Even though five decades have passed, no laboratory gold standard is available and like all clinical entities which are diagnosed exclusively by presence of clinical signs, KD too is vulnerable to underestimation depending upon the specificity of signs used for its identification.
\end{abstract}

Keywords: Sepsis; Kawasaki disease; Infant

\section{Introduction}

Despite advances in the antimicrobial therapy and implemen-

Manuscript accepted for publication March 31, 2014

${ }^{a}$ Department of Pediatrics, SUNY Downstate Medical Center, 450 Clarkson Avenue, Brooklyn, NY 11203, USA

${ }^{b}$ Department of Pediatric Cardiology, SUNY Downstate Medical Center, 450 Clarkson Avenue, Brooklyn, NY 11203, USA

${ }^{c}$ Department of Pediatric Infectious Diseases, SUNY Downstate Medical

Center, 450 Clarkson Avenue, Brooklyn, NY 11203, USA

${ }^{\mathrm{d}}$ Corresponding Author: Suraj Pratap, 131, 74th Street, Apt 3B,

Brooklyn, NY 11209, USA. Email: pratap.suraj@gmail.com

doi: http://dx.doi.org/10.14740/ijcp147w tation of a multitude of screening protocols, group B streptococcus (GBS) infection remains a major cause of morbidity and mortality in newborns and young infants. Here we present a case of GBS sepsis in a 3-month-old child with cardiac findings so striking that the medical team caring for the child was forced to digress from the established criteria for Kawasaki disease (KD). KD is one of the most common causes of coronary vasculitis in young children, and there is up to $25 \%$ chance of serious cardiovascular damage if treatment is not given early in the course of disease [1]. Since no laboratory gold standard is available, the diagnosis of $\mathrm{KD}$ depends upon the specificity of signs used for its identification. It is not surprising therefore, that the diagnostic criterions of $\mathrm{KD}$ have been a topic of constant debate.

\section{Case Report}

An 87-day-old girl was brought to our emergency room (ER) with fever, tachypnea, tachycardia and a perineal rash of 1 day duration that was erythematous, non-palpable and nontender. In the ER she had a temperature of $102.2^{\circ} \mathrm{F}$, heart rate of $200-210$ beats per minute (bpm) and respiratory rate of 40 - 60 breaths per minute. About 3 days prior, her parents noticed that she felt warm and upon measurement her otic temperature was just above $99^{\circ} \mathrm{F}$. A day prior to admission she had developed a fever of $100.5^{\circ} \mathrm{F}$ at home, was breathing faster than usual and appeared more tired. Initial exam and laboratory investigations in the ER did not reveal any obvious source of infection. The patients past medical history and family history were non-contributory. The comprehensive metabolic panel, chest X-ray and urinalysis were essentially normal while a complete blood count $(\mathrm{CBC})$ showed mild thrombocytosis (WBC $10.9 \times 10^{3} / \mu \mathrm{L}, \mathrm{Hb} 11 \mathrm{~g} / \mathrm{dL}$ and platelet count $\left.450 \times 10^{3} / \mu \mathrm{L}\right)$. The $\mathrm{C}$-reactive protein $(\mathrm{CRP})$ was found to be $71 \mathrm{mg} / \mathrm{dL}$ and samples were sent for blood and urine culturing. She was given a fluid bolus, antipyretics and intravenous (IV) ceftriaxone for empiric coverage.

On the pediatric floor she continued to be persistently tachycardic (200 - $210 \mathrm{bpm})$ despite rehydration and fever control. The EKG showed sinus tachycardia and she was transferred to the pediatric ICU for closer monitoring. The 


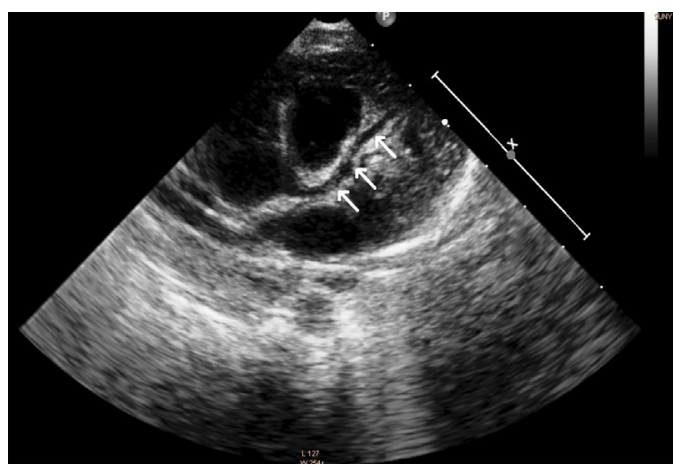

Figure 1. Echocardiogram of the patient showing dilated LAD coronary artery during the acute phase of illness.

echocardiogram showed significant dilatation of left main coronary artery (LMCA) and left anterior descending (LAD) coronary artery along with perivascular brightness and lack of tapering suggestive of vascular inflammation (Fig. 1). The vascular dimensions were measured in a conservative manner and the $\mathrm{z}$ scores were found to be 1.1 and 2.66 respectively for the LMCA and LAD coronary artery.

On the second hospital day patient showed clinical improvement and her vitals stabilized except a persistently elevated heart rate. Her blood culture grew Gram-positive cocci which were identified as GBS on the next day. A repeat echocardiogram performed by another pediatric cardiologist confirmed the findings of the first report and obtained identical values upon the calculation of $\mathrm{z}$ scores. Having managed many pediatric patients with KD in the past, the cardiology team was highly suspicious of a concomitant vasculitis which could possibly be a sign of a recently missed or an ongoing $\mathrm{KD}$. Blood counts and inflammatory markers were repeated while rapid plasma reagin, HIV serology, cell surface markers and immunoglobulin levels were also sent to rule out any infectious or immune-mediated disease. Repeat CBC showed $20.6 \times 10^{3} / \mu \mathrm{L}$ WBC, $9.2 \mathrm{~g} / \mathrm{dL} \mathrm{Hb}$ and $373 \times$ $10^{3} / \mu \mathrm{L}$ platelets (manual differential count: $64 \%$ segmented cells, 7\% bands and 21\% lymphocytes). The second CRP was $96 \mathrm{mg} / \mathrm{dL}$ and other test results were within acceptable range. The patient was treated with $2 \mathrm{~g} / \mathrm{kg}$ of IV immunoglobulin $\mathrm{G}$ and high dose aspirin with a presumed diagnosis of incomplete KD. Patient also completed a 14-day course of IV antibiotics in the hospital. Follow-up investigations done before discharging the patient showed improvement in coronary vasculitis and a normalized CRP $(<4 \mathrm{mg} / \mathrm{dL})$.

\section{KD in Young Infants: A Clinical Enigma}

Classically KD has been defined by a fever of 5 or more days along with at least four of the other principal features (changes in extremity, polymorphous rash, bilateral bulbar conjunctivitis, changes in lip or oral cavity and cervical lymphade- nopathy) [2, 3]. However, there have been an increasing number of incidences where all the major features are not met and patients are diagnosed with so-called "atypical" or "incomplete" KD and treated to prevent coronary complications [4]. The term incomplete is preferred over atypical, because these patients do not differ much from those with classical KD except that they do not satisfy the criteria to fulfill the epidemiological case definition. This happens primarily because the symptoms are distributed more widely in time [5]. Other causes of vasculitis in infantile age group include rheumatic fever, henoch-schonlein purpura, polyarteritis nodosa (PAN), Takayasu disease and systemic lupus erythematosus. Isolated vasculitis of the coronary arteries, however, is much less common and it is seen mostly in association with $\mathrm{KD}$ or rarely with PAN.

Most of the features of KD are an outcome of widespread inflammation of medium- and small-sized blood vessels, and its diagnosis therefore is based upon manifestations of systemic inflammation. The characteristic findings, which primarily include fever, muco-cutaneous changes and lymphadenopathy, are often preceded by a brief respiratory or gastrointestinal prodrome [6]. Even though these clinical signs form the basis for the diagnostic criteria for $\mathrm{KD}$, it is important to keep in mind that they are often not present at the same time. Also the specificity of neither of these findings nor the collective conglomerate is anywhere near $100 \%$, and hence children who do not meet the criteria may have an incomplete form of KD. Studies have shown that around $10 \%$ of children who develop coronary artery aneurysms never meet criteria for KD [7]. Conversely, some patients who have five or six signs of KD may actually have some other medical conditions.

Fever is the most consistent manifestation of KD and it reflects an elevation of pro-inflammatory cytokines, which mediate the underlying vascular inflammation. Fever of KD is minimally responsive to antipyretic agents and antibiotics, and it typically remains above $38.5^{\circ} \mathrm{C}$ during most of the illness. However the use of specific fever duration as a rigid criterion has often been questioned especially in recent years, since the functional and structural cardiac changes can now be more readily assessed due to easy availability of echocardiogram. There are documented case reports where infants developed florid symptoms and complications of KD in absence of fever or after an initial favorable response to antibiotics as evidenced by resolution of fever [8-10]. More importantly, the first guidelines were published by Dr. Kawasaki before cardiac involvement was recognized in this disease, and hence they were never intended to identify children at risk for developing coronary artery abnormalities.

The diagnosis of incomplete KD is problematic because it rests upon clinical judgment and supportive laboratory findings, but remains uncertain unless the child develops coronary complications. The incidence of incomplete KD is not known but studies have suggested that it is higher for 
infants younger than 6 months $[10,11]$. Infants are also at increased risk of coronary artery aneurysms, probably because of the delay in treatment as they often do not meet the complete diagnostic criteria [12].

Apart from clinical features there are other parameters such as laboratory tests (acute phase reactants, hepatic enzymes, leukocytes and platelets) and echocardiogram findings which can be used to support the diagnosis of an incomplete $\mathrm{KD}$. The echocardiogram is a useful diagnostic tool and is considered positive if any of three conditions are met: $\mathrm{z}$ score of LAD or RCA $\geq 2.5$, coronary arteries meet Japanese Ministry of Health criteria for aneurysms, or $\geq 3$ other suggestive features exist (perivascular brightness, lack of tapering, decreased LV function, mitral regurgitation, pericardial effusion, or z scores in LAD or RCA of 2 - 2.5). However, none of these findings, including coronary artery dilatation, is pathognomonic for KD. A prospective study of echocardiograms in febrile children found that conditions other than $\mathrm{KD}$ can be associated with some degree of coronary artery dilatation. However, only children with KD had coronary artery $\mathrm{z}$ scores more than or equal to 2.5 , suggesting that significant coronary artery enlargement is indeed a reliable marker of $\mathrm{KD}$ [13].

In this instance the patient presented with fever and rash, but she did not fit into the classical diagnostic pentad of KD. Notable laboratory findings included lecukocytosis, thrombocytosis and an elevated CRP which were present at different times during the course of illness. The initial impression was that of an obvious infectious or inflammatory process but the subsequent cardiac findings radically changed the course of management.

This case elucidates the deficiencies in our current understanding of $\mathrm{KD}$ in young infants and the need for better investigative techniques that may help us confidently diagnose this acute condition. As with many cases of KD in young infants, the management in this case too was not ideal, and plausible arguments can be cited in favor or against the individual therapeutic interventions and their timings. Many questions still remain unanswered and should encourage thoughtful discussions. For instance, can an acute bacterial or viral infection (like GBS in this case) trigger the chain of events which may lead to systemic inflammation and vasculitis indistinguishable from KD? In absence of a 5-day long fever, can we use rash, echocardiographic findings and lab markers of inflammation for a presumptive diagnosis of $\mathrm{KD}$ in young infants? Considering the high cardiovascular morbidity in this age group, how long should we wait to administer IV immunoglobulin once significant coronary changes have been identified in these patients?

This patient reminded us that although precise clinical definitions of many disease entities are available in the literature, an enormous part of decision making in pediatric medicine actually depends on experience and clinical wisdom. It is important to realize that medicine is an inexact science, and despite our attempts to streamline the definitions of illnesses and bring pathology to the realm of statistics there is a vastness that cannot be rationalized.

\section{Acknowledgement}

We express our gratitude to the residents and nurses working in the pediatric ward and ICU of SUNY Downstate Medical Center. We also thank Dr. Arun and Dr. Dhuper from the Departments of Pediatric Infectious Diseases and Pediatric Cardiology respectively for their valuable inputs. Thanks are also due to Dr. Doymez, Dr. Braimen and Dr. Schlomowich who were directly involved in patient care.

\section{References}

1. Burns JC, Glode MP. Kawasaki syndrome. Lancet. 2004;364(9433):533-544.

2. Dajani AS, Taubert KA, Gerber MA, Shulman ST, Ferrieri $\mathrm{P}$, Freed $\mathrm{M}$, Takahashi $\mathrm{M}$, et al. Diagnosis and therapy of Kawasaki disease in children. Circulation. 1993;87(5):1776-1780.

3. Ayusawa M, Sonobe T, Uemura S, Ogawa S, Nakamura Y, Kiyosawa N, Ishii M, et al. Revision of diagnostic guidelines for Kawasaki disease (the 5 th revised edition). Pediatr Int. 2005;47(2):232-234.

4. Witt MT, Minich LL, Bohnsack JF, Young PC. Kawasaki disease: more patients are being diagnosed who do not meet American Heart Association criteria. Pediatrics. 1999;104(1):e10.

5. Newburger JW, Takahashi M, Gerber MA, Gewitz MH, Tani LY, Burns JC, Shulman ST, et al. Diagnosis, treatment, and long-term management of Kawasaki disease: a statement for health professionals from the Committee on Rheumatic Fever, Endocarditis and Kawasaki Disease, Council on Cardiovascular Disease in the Young, American Heart Association. Circulation. 2004;110(17):2747-2771.

6. Huang GY, Ma XJ, Huang M, Chen SB, Huang MR, Gui YH, Ning SB, et al. Epidemiologic pictures of Kawasaki disease in Shanghai from 1998 through 2002. J Epidemiol. 2006;16(1):9-14.

7. Sundel RP. Update on the treatment of Kawasaki disease in childhood. Curr Rheumatol Rep. 2002;4(6):474-482.

8. Maresi E, Passantino R, Midulla R, Ottoveggio G, Orlando E, Becchina G, Meschis L, et al. Sudden infant death caused by a ruptured coronary aneurysm during acute phase of atypical Kawasaki disease. Hum Pathol. 2001;32(12):1407-1409.

9. Hinze CH, Graham TB, Sutherell JS. Kawasaki disease without fever. Pediatr Infect Dis J. 2009;28(10):927- 
928.

10. Dodi I, Raggi V, Verna M, Tchana B, Vignali D, Bandello MA, Lacava S, et al. Atipical Kawasaki disease with coronary aneurysm in infant. Ital J Pediatr. 2011;37:19.

11. Joffe A, Kabani A, Jadavji T. Atypical and complicated Kawasaki disease in infants. Do we need criteria? West J Med. 1995;162(4):322-327.

12. Chang FY, Hwang B, Chen SJ, Lee PC, Meng CC,
Lu JH. Characteristics of Kawasaki disease in infants younger than six months of age. Pediatr Infect Dis J. 2006;25(3):241-244.

13. Bratincsak A, Reddy VD, Purohit PJ, Tremoulet AH, Molkara DP, Frazer JR, Dyar D, et al. Coronary artery dilation in acute Kawasaki disease and acute illnesses associated with Fever. Pediatr Infect Dis J. 2012;31(9):924-926. 